\title{
A feature positive effect in conditioned suppression
}

\author{
DOUGLAS REBERG and ROBERT LECLERC \\ University of Western Ontario, London, Ontario N6A 5C2, Canada
}

\begin{abstract}
A conditioned suppression experiment with rats studied the development of two discriminations involving two conditioned stimuli, $A$ and $X$. In one discrimination ( $\mathrm{X}+/ \mathrm{A}-$ ), compound presentations of $A$ and $X$ signaled shock and presentations of $A$ alone signaled no-shock. In the other discrimination $(\mathrm{A}+/ \mathrm{AX}-)$, A alone signaled shock and AX signaled no-shock. AX+/A- discriminations were learned more rapidly than their A+/AX - counterparts. These results, which resemble the featurepositive effect of Jenkins and Sainsbury $(1969,1970)$, are discussed in terms of Rescorla and Wagner's (1972) theory of conditioning and also in terms of stimulus intensity mechanisms.
\end{abstract}

Jenkins and Sainsbury $(1969,1970$; see also Jenkins, 1973) have conducted several experiments in pigeon discrimination training with "feature positive" and "feature negative" procedures. A typical experiment involves two visual displays. One (display A) might be a lighted pigeon key, the other (AX) a lighted key with an added visual feature, such as a small dot. AX $+/ \mathrm{A}-$ discriminations (where responses to $\mathrm{AX}$ are reinforced but responses to $\mathrm{A}$ are not) are said to be "feature positive." $\mathrm{A}+/ \mathrm{AX}-$ discriminations (where responses to $A$ are reinforced and responses to $\mathrm{AX}$ are not) are said to be "feature negative."

Several interesting phenomena are associated with these discriminations, including the "feature tracking" effects recently reviewed by Hearst and Jenkins (1974) and by Hearst (1975). The effect of interest for this paper is Jenkins and Sainsbury's finding that feature-positive discriminations are easier for pigeons to master than feature-negative discriminations. As a rule, pigeons easily learn to respond to $\mathrm{AX}$ but not to $A$ in feature-positive training, but they usually continue to respond indiscriminately in featurenegative discriminations despite extensive training. This difference between the procedures has become known as the "feature-positive effect." Similar results have also been reported in "autoshaped" Pavlovian discriminations (Hearst, 1975), children's discrimination learning (Sainsbury, 1971), and conditioned taste aversion discriminations in rats (cf., the SAC + vs. SAC - discriminations in Rusiniak, Garcia, \& Hankins, 1976, Experiment 2).

Parallels of feature-negative discriminations have

This research was supported by NRC Grant A-9588 to Douglas Reberg. We are grateful to John Memmott, who made valuable comments on an earlier version of this paper. Requests for reprints should be sent to D. Reberg at the Department of Psychology, University of Western Ontario, London, Ontario N6A 5C2, Canada. been studied for many years in Pavlovian laboratories under the heading of "conditioned inhibition training." An early salivary conditioning experiment by Pavlov (1927), for example, used two conditioned stimuli, A (a rotating object) and X (a tactile stimulus). On some trials, A was presented alone and followed by food. On other trials, $\mathrm{A}$ and $\mathrm{X}$ were presented simultaneously (forming a compound $\mathrm{AX}$ ) and no food followed. The resulting $A+/ A X-$ paradigm is similar to a feature-negative discrimination where $A$ has a role analogous to the lighted key and $X$ has a role analogous to the visual feature. As Pavlov and many others (cf. Rescorla, 1969) have confirmed, the result of $\mathbf{A}+/ \mathbf{A X}-$ training in Pavlovian discriminations is a conditioned response elicited by $\mathrm{A}$ but not by $\mathrm{AX}$. The comparative absence of responding to $\mathrm{AX}$ is generally attributed to inhibitory properties acquired by $\mathrm{X}$ that interfere with the ability of $\mathrm{A}$ to elicit the conditioned response.

Pavlov did not specifically study the $\mathrm{AX}+/ \mathrm{A}-$ paradigm that parallels feature positive discriminations, and there are few available data that permit direct comparison of $\mathbf{A}+/ \mathbf{A X}-$ and $\mathbf{A X}+/ \mathbf{A}-$ Pavlovian discriminations. The present experiment compared the development of $A+/ A X$ - and $\mathrm{AX}+/ \mathrm{A}-$ discriminations in conditioned suppression procedures with rats. Our objective was to determine if $\mathbf{A X}+/ \mathbf{A}-$ discriminations develop more rapidly than their $\mathbf{A}+/ \mathbf{A X}$ - counterparts in a Pavlovian situation where $\mathrm{A}$ and $\mathrm{X}$ are visual or auditory stimuli differentially associated with shock.

\section{METHOD}

\section{Subjects}

The subjects were 16 male hooded rats purchased from the Canadian Breeding Laboratory, St. Constant, Quebec. The rats were individually caged and deprived to $80 \%$ of ad-lib body weights.

\section{Apparatus}

Four identical experimental chambers $(24.1 \times 22.9 \times$ 
$30.3 \mathrm{~cm}$ ) were used. Each chamber had Plexiglas side walls and top and stainless steel end panels. The floor was a grid of 19 stainless steel rods, through which scrambled shock (1.3 mA, $.5 \mathrm{sec}$ ) could be delivered from individual Grason-Stadler Model 700 shock generators.

Centered on one wall $9.1 \mathrm{~cm}$ from the floor was a rat lever, $5.1 \times 1.2 \times 1.5 \mathrm{~cm}$, that required a pressure of about $20 \mathrm{~g}$ for activation. In the lower left corner of the same wall was a small food cup into which $45-\mathrm{mg}$ Noyes food pellets could be delivered. Mounted outside each box was a $10.2-\mathrm{cm}$ speaker positioned directly overhead, a $7-W$ houselight (on throughout each session), and a $25-\mathrm{W}$ refrigerator bulb. Each box was enclosed in a light-tight sound-attenuated chamber.

The conditioned stimuli were intermittent tone and flashing light. The tone was $1,000 \mathrm{~Hz}$ and $75 \mathrm{~dB}$ (re: .0002 dynes $/ \mathrm{cm}^{2}$ ) interrupted 10 times $/ \mathrm{sec}$. The light was provided by the $25-\mathrm{W}$ refrigerator bulb, which flashed off briefly once per second.

\section{Procedure}

Barpress training and pretest. On the first day of training, food pellets were delivered to each subject on a VI 30-sec responseindependent schedule. Additionally, every barpress was reinforced by a single pellet. This procedure continued until $60 \mathrm{~min}$ had elapsed or at least $\mathbf{4 0}$ barpresses were recorded. A few rats required a second session to begin barpressing.

After preliminary barpress training, the rats were divided into four groups of four. Each group received 1-h session in which barpresses were reinforced on a VI 30-sec schedule, followed by seven 1 -h sessions in which barpresses were reinforced on a VI 1 -min schedule.

Pretests of the stimuli to be used in conditioning were made in the next two sessions. While the rat barpressed on the VI 1-min schedule, three $60-\mathrm{sec}$ presentations of conditioned stimulus $\mathrm{A}$ and three 60 -sec presentations of the compound stimulus AX were scheduled in a random sequence. For Groups 1 and 3, stimulus $A$ was flashing light (L) and $X$ was intermittent tone (T). For Groups 2 and 4, the stimuli were reversed. The AX compound was a simultaneous presentation of both stimuli with common onset and offset.

Conditioning. Conditioned suppression training took place in the next 32 1-h barpress sessions. Each session included three 60-sec presentations of $\mathrm{A}$ and three 60-sec presentations of $\mathrm{AX}$, scheduled in a random sequence. Groups $1(\mathrm{LT}+/ \mathrm{L}-)$ and 2 $(\mathrm{TL}+/ \mathrm{T}-)$ were trained with an $\mathrm{AX}+/ \mathrm{A}-$ discrimination procedure, where presentations of AX terminated with a $1.3-\mathrm{mA} .5-\mathrm{sec}$ shock and presentations of $A$ never terminated with shock. Groups $3(\mathrm{~L}+/ \mathrm{LT}-)$ and $4(\mathrm{~T}+/ \mathrm{TL}-)$ were trained with $\mathbf{A}+/ \mathbf{A X}-$ procedures, where presentations of $\mathbf{A}$ terminated with shock and presentations of AX never terminated with shock.

\section{RESULTS}

Suppression of barpressing for each rat during A and $\mathbf{A X}$ was tabulated in terms of suppression ratios, $b /(a+b)$, where $a=$ the mean number of barpresses that occurred during the six 60 -sec intervals that immediately preceded stimulus presentations, and $b=$ the mean number of barpresses that occurred during the three presentations of A or AX. A strong suppressing effect of a stimulus is indicated by suppression ratios approaching 0 , while weaker suppression is indicated by suppression ratios approaching .50.

Figure 1 shows the results for all groups plotted as mean suppression ratios over blocks of conditioning days. The individual data points at the left of each panel show pretest suppression ratios for $A$ (open circles) and AX (filled circles) averaged over the 2 pretest days. The joined points in each panel show $\mathrm{A}$ and $\mathrm{AX}$ suppression ratios in conditioning, averaged over blocks of 4 days.

\section{Pretest}

None of the stimuli produced substantial suppression in pretest. Presentations of AX generally produced more suppression than presentations of $A$, and pretest presentations of $L$ (in Groups 1 and 3) generally produced more suppression than $T$ (in Groups 2 and 4). A 2 by 2 by 2 analysis of variance with Discriminations $(\mathrm{AX}+/ \mathrm{A}-$ vs. $\mathrm{A}+/ \mathrm{AX}-)$, Stimuli $(A=L \quad X=T$ vs. $A=T \quad X=L)$, and $C S s$ (AX vs. A) as factors confirmed this general pattern by indicating significant effects of CSs $[F(1,28)=$ $7.07, \mathrm{p}<.05]$ and a significant Stimuli by CSs interaction $[\mathrm{F}(1,28)=5.49, \mathrm{p}<.05]$.

\section{Acquisition of the Discrimination}

Inspection of Figure 1 indicates that there were only minor differences among groups in suppression on shock-reinforced trials, but that there were clear differences among groups in the rapidity and degree to which suppression was eliminated on unreinforced trials. Discrimination training proceeded most rapidly in Groups 1 and 2, both trained with the $\mathrm{AX}+/ \mathrm{A}-$ procedure. Groups 3 and 4, trained with the $\mathrm{A}+/ \mathrm{AX}$ - procedure, were much slower to acquire the discrimination. The $\mathrm{AX}+/ \mathrm{A}-$ discrimination developed slightly more rapidly in Group $1(\mathrm{LT}+/ \mathrm{T}-)$ than in Group $2(\mathrm{TL}+/ \mathrm{T}-)$. The $\mathrm{A}+/ \mathrm{AX}-$ discrimination developed much more rapidly in Group $4(\mathrm{~T}+/ \mathrm{TL}-)$ than in Group $3(\mathrm{~L}+/ \mathrm{LT}-)$. Group 3 did not show an appreciable difference in suppression on reinforced and unreinforced trials until the last conditioning block.

Separate analyses of variance for reinforced and unreinforced trials generally confirmed the results indicated by Figure 1. The suppression ratios for shock-reinforced trials (AX + for Groups 1 and 2, $A+$ for Groups 3 and 4) were submitted to a 2 by 2 by 8 analysis of variance with Discriminations $(A X+/ A-$ vs. $A+/ A X-)$, Stimuli $(A=L X=T$ vs. $A=T T=L$ ), and Conditioning Blocks as factors. The analysis confirmed a significant effect of Blocks $[F(7,84)=20.13 ; p<.01]$, and also indicated a significant Blocks by Stimuli interaction $[F(7,84)=$ $2.25 ; \mathrm{p}<.05$ ]. Newman-Keuls comparisons traced the interaction to Block 1 , where $\mathrm{AX}+$ and $\mathrm{A}+$ suppression ratios for Groups 2 and 4 were significantly smaller than those for Groups 1 and 3, indicating stronger suppression on shock-reinforced trials in the first block when $\mathrm{A}=$ tone and $\mathrm{X}=$ light (ps < .05). There were no significant differences in suppression on shock-reinforced trials at any other point in training.

Suppression ratios for the unreinforced trials 
$A=L ! G H T X=T O N E$

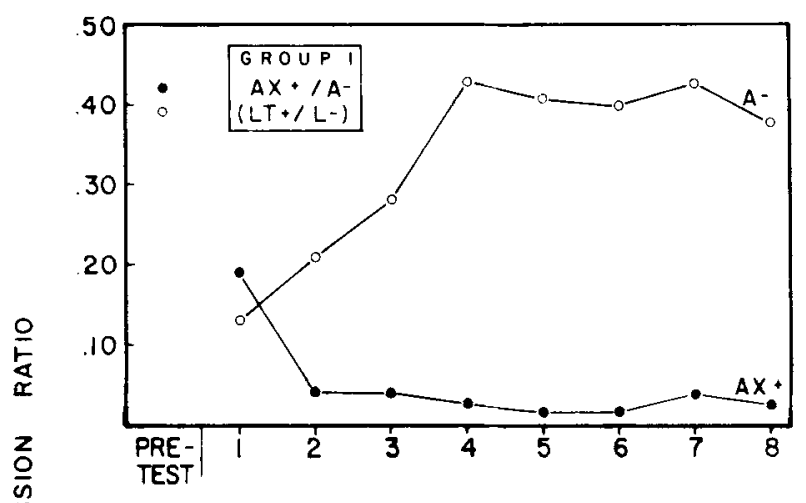

$A=T O N E X=L I G H T$
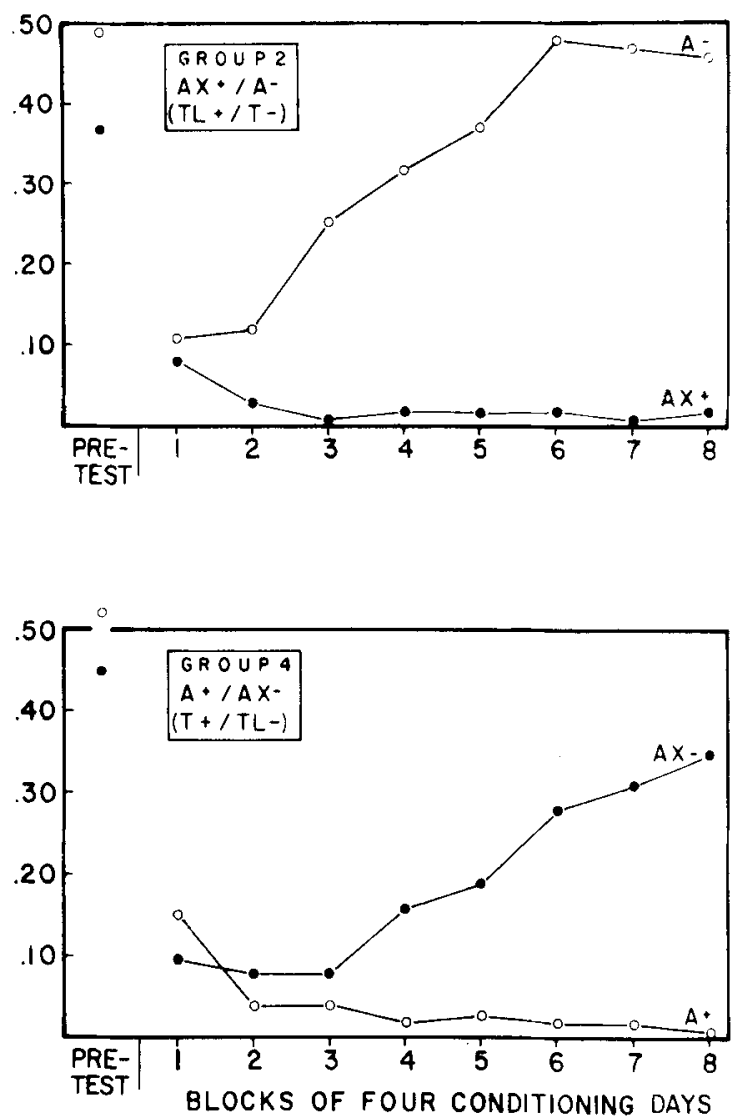

Figure 1. Pretest and conditioning results for all groups in terms of suppression ratios as a function of blocks of sessions.

(A - for Groups 1 and 2, AX - for Groups 3 and 4) were submitted to a similar 2 by 2 by 8 analysis of variance, with Discriminations, Stimuli, and Conditioning Blocks as factors. The analysis indicated significant main effects of Blocks $[F(7,84)=17.76$, $\mathrm{p}<.01]$ and Discriminations $[\mathrm{F}(1,12)=16.93$, $\mathrm{p}<.01]$, and significant interactions of Discriminations by Blocks $[F(7,84)=6.46 ; p<.01]$, Stimuli by Blocks $[F(7,84)=3.70 ; p<.01]$, and Discriminations by Stimuli by Blocks $[F(7,84)=3.29$; $\mathrm{p}<.01]$. Newman-Keuls comparisons for individual conditioning blocks confirmed the general pattern that is evident from Figure 1. In Blocks 1 and 2, there were no significant differences among groups in suppression to $\mathbf{A}-$ or $\mathbf{A X}-$. In Blocks 3 through 7, A - suppression ratios for Groups 1 . and 2 were significantly larger than $\mathrm{AX}$ - ratios for Groups 3 and 4, and in Blocks 4 through 7, AXratios for Group 4 were significantly larger than AX - ratios for Group 3. Finally, in Block 8, there were no significant differences among $A-$ ratios in Groups 1 and 2 and $A X$ - ratios for Group 4, but each was significantly larger than the $A X-$ ratios for Group 3. Although Figure 1 indicates that $\mathbf{A}$ - suppression was eliminated slightly more rapidly in Group 1 than in Group 2, those differences were not statistically significant at any point in training ( $\mathrm{ps}>.05)$.

\section{DISCUSSION}

Rats mastered conditioned suppression discriminations more rapidly with $\mathbf{A X}+/ \mathbf{A}-$ than with $A+/ A X-$ training. These results are similar to the differences between the feature-positive and featurenegative discriminations that Jenkins and Sainsbury $(1969,1970)$ reported in operant conditioning with pigeons, and indicate that conditioned suppression should be included among the procedures that produce feature-positive effects. There are potentially important differences between the pigeon and rat experiments; for instance, pigeons virtually never master $\mathrm{A}+/ \mathrm{AX}$ - feature-negative discriminations, in contrast to the present experiment, where all subjects made at least some progress towards mastering the $\mathrm{A}+/ \mathbf{A X}$ - discrimination. Nevertheless, it is clear that the advantages of $\mathrm{AX}+/ \mathrm{A}-$ over $\mathrm{A}+/ \mathrm{AX}-$ training are not limited to operant discriminations involving pigeons, localized visual displays, and food reinforcement. 
The feature-postive effect is in agreement with predictions of Rescorla and Wagner's (1972) theory of conditioning. The theory essentially follows Pavlov's (1927) analysis of $\mathrm{A}+/ \mathrm{AX}$ - discriminations by emphasizing the opponent roles of conditioned excitation and conditioned inhibition. According to the Rescorla-Wagner theory, A+ trials result in A developing excitatory strength (which, in the present experiment, would be indicated by the development of suppression). Initially, the excitatory strength of $\mathrm{A}$ also dominates the $\mathrm{AX}$ compound. With continued $A+/ A X$ - training, however, $X$ develops inhibitory strength that opposes $A$, thus eliminating excitatory effects in the $\mathbf{A X}$ compound.

The theory indicates a different set of mechanisms for $\mathbf{A X}+/ \mathbf{A}-$ discriminations. Early in $\mathbf{A X}+/ \mathbf{A}-$ training, $A$ and $X$ each develop some excitatory strength as they "share" the effects of $\mathrm{AX}+$ trials. However, according to the theory, since $\mathrm{X}$ is only present on reinforced $\mathrm{AX}+$ trials while $\mathrm{A}$ is present on both reinforced $\mathbf{A X}+$ trials and unreinforced A - trials, $\mathrm{X}$ eventually becomes a strong excitatory stimulus that accounts for practically all of the strength of the AX compound, while A loses most of its excitatory strength and becomes relatively neutral, thus eliminating excitatory effects on $\mathrm{A}$ - trials. Although other distinctions between $\mathrm{A}+/ \mathrm{AX}-$ and $\mathrm{AX}+/ \mathrm{A}-$ training may be made on the basis of the Rescorla-Wagner theory (see Jenkins, 1973), the theory's major point is that $\mathrm{AX}+/ \mathrm{A}-$ discriminations do not require $A$ to develop inhibitory strength to eliminate excitatory effects on $A-$ trials. $\mathrm{AX}+/ \mathrm{A}$ - discriminations thus develop more rapidly because they require less extensive changes in associative strength of the stimulus that signals nonreinforcement.

In the present experiment, $\mathbf{A}+/ \mathbf{A X}-$ discriminations developed more rapidly in Group $4(\mathrm{~T}+/ \mathrm{TL}-)$ than in Group $3(\mathrm{~L}+\mathrm{LT}-)$. The Rescorla-Wagner theory deals with such differences in terms of stimulus "salience" parameters, indicating that certain stimuli develop associative strength more rapidly than others. The theory might argue, for example, that $\mathrm{L}$ was a more salient stimulus than $\mathrm{T}$. The rapid development of the $\mathrm{T}+/ \mathrm{TL}$ - discrimination in Group 4 could then be attributed to $L$ being a salient stimulus that developed inhibitory strength quickly, and the slower $\mathrm{L}+/ \mathrm{LT}$ - discrimination in Group 3 could be attributed to $T$ being a less salient stimulus that was slower to develop inhibitory strength.

However, from that interpretation one would also expect to find similar differences between Groups $1(\mathrm{LT}+/ \mathrm{L}-)$ and $2(\mathrm{TL}+/ \mathrm{T}-)$. A more salient $\mathrm{L}$ should have absorbed a greater "share" of the effects of LT + trials in Group 1 and $T L+$ trials in Group 2, resulting in $\mathrm{L}$ developing more excitatory strength than T. This "overshadowing" mechanism (Kamin, 1969), in turn, should have favored rapid development of the $\mathrm{TL}+/ \mathrm{T}$ - discrimination in Group 2 and slower development of the $\mathrm{LT}+/ \mathrm{L}$ - discrimination in Group 1. The present results are, if anything, contrary to that expectation, since the $\mathrm{LT}+/ \mathrm{L}$ - discrimination in Group 1 developed slightly (but not significantly) faster than the $\mathrm{TL}+/ \mathrm{T}$ - discrimination in Group 2.

One interpretation of these data that could be incorporated into Rescorla and Wagner's theory is that $\mathrm{L}$ is a more salient stimulus than $\mathrm{T}$ for distinguishing no-shock trials in $\mathrm{A}+/ \mathrm{AX}$ - training, and that $\mathrm{L}$ and $T$ are about equally salient (with $T$ perhaps slightly more salient than $\mathrm{L}$ ) as stimuli for distinguishing shock trials in $\mathrm{AX}+/ \mathrm{A}-$ training. Although we cannot be certain of the accuracy of that interpretation in the absence of further experiments, such interactions between stimuli and the events they signal might not be surprising, given the abundance of evidence for stimulus-reinforcer specificity in other experimental procedures (e.g., Foree \& LoLordo, 1973; Garcia \& Koelling, 1966; LoLordo \& Furrow, 1976).

Although Rescorla and Wagner's theory provides a general framework for describing the development of A vs. AX discrimination, it is possible that processes not considered by the theory are also involved. For example, it may be appropriate to regard some A vs. AX discriminations as involving two stimuli of different intensities, where $\mathrm{AX}$ is more intense than A. Several experiments have demonstrated that intensity discriminations (such as soft tone vs. loud tone or dim light vs. bright light) are usually acquired more rapidly when the more intense stimulus signals reinforcement (D'Amato \& Jagoda, 1961; Pierrel, Sherman, Blue, \& Hegge, 1970; Sadowsky, 1966). Such results have led Pierrel et al. (1970) to propose that discrimination training may generally be facilitated by arranging for the more intense stimulus to signal reinforcement. Perhaps such a principle contributes to the more rapid development of many $\mathbf{A X}+/ \mathrm{A}-$ discriminations, where the stimulus that is presumably more intense signals reinforcement.

One would not anticipate that stimulus intensity mechanisms would be important in all $A$ vs. AX discriminations, since feature-positive effects have often been demonstrated in situations where it would be difficult to argue that $\mathrm{A}$ and $\mathrm{AX}$ differed appreciably in physical intensity (e.g., the "three key" displays used by Hearst, 1975, where A might be three pigeon keys illuminated green, AX two keys illuminated green and a third illuminated red). Such intensity effects deserve further study in $A$ vs. $A X$ discriminations in conditioned suppression, however, where the importance of conditioned stimulus intensity has been well documented (Kamin, 1965). 
Particularly careful investigation will be necessary to determine the extent to which similar mechanisms influence feature-positive effects obtained with other combinations of stimuli, responses, and reinforcers.

\section{REFERENCES}

D'Amato, M. R., \& JaGoda, H. Studies of transfer of brightness discrimination from bar-pressing to a $\mathrm{Y}$ maze. Joumal of Psychology, 1961, 52, 411.422.

FOREE, D. D. \& LoLoRDo, V. M. Attention in the pigeon: Differential effects of food-getting vs. shock-avoidance procedures. Journal of Comparative and Physiological Psychology, 1973. 85, 551-558.

Garcia. J., \& Koelling, R. A. Relation of cue to consequence in avoidance learning. Psychonomic Science, 1966, 4, 123-124.

HeARST, E. Pavlovian conditioning and directed movements. In G. H. Bower (Ed.). The psychology of leaming and motivation (Vol. 9). New York: Academic Press, 1975.

Hearst, E., \& Jenkins, H. M. Sign-tracking: The stimulusreinforcer relation and directed action. Austin. Texas: Psychonomic Society, 1974.

JenkINS, H. M. Noticing and responding in a discrimination based on a distinguishing element. Learning and Motivation, $1973,4,115 \cdot 137$.

Jenkins, H. M., \& Sainsbury, R. S. The development of stimulus control through differential reinforcement. In $\mathbf{N}$. J. Mackintosh \& W. K. Honig (Eds.), Fundamental issues in associative learning. Halifax: Dalhousie University Press, 1969.

Jenkins. H. M., \& Sainsbury, R. S. Discrimination learning with the distinctive feature on positive or negative trials. In D. I. Mostofsky (Ed.), Attention: Contemporary theory and analysis. New York: Appleton-Century-Crofts, 1970.

Kamin, L. J. Temporal and intensity characteristics of the conditioned stimulus. In W. F. Prokasy (Ed.), Classical conditioning: A symposium. New York: Appleton-Century-Crofts, 1965.

Kamin, L. J. Predictability, surprise, attention, and conditioning. In B. A. Campbell \& R. M. Church (Eds.), Punishment and aversive behavior. New York: Appleton-Century-Crofts, 1969.

LoLordo, V. M., \& FurRow, D. R. Control by the auditory or the visual element of a compound discriminative stimulus: Effects of feedback. Journal of the Experimental Analysis of Behavior, 1976, 25, 251-256.

Pavlov, I. P. Conditioned reflexes. New York: Dover, 1927.

Pierrel, R., Sherman, J. G., Blue, S., \& Hegge, F. W Auditory discrimination: A three-variable analysis of intensity effects. Journal of the Experimental Analysis of Behavior, $1970,13,17-35$

Rescorla, R. A. Pavlovian conditioned inhibition. Psychological Bulletin, 1969, 72, 77-94.

Rescorla, R. A., \& WAGNer, A. R. A theory of Pavlovian conditioning: Variations in the effectiveness of reinforcement or nonreinforcement. In A. H. Black \& W. F. Prokasy (Eds.), Classical conditioning II: Current research and theory. New York: Appleton-Century-Crofts, 1972.

Rusiniak, K. W., Garcia, J., \& Hankins, W. G. Bait shyness: Avoidance of the taste without escape from the illness. Journal of Comparative and Physiological Psychology. $1976,90,460-467$

SADowsky, S. Discrimination learning as a function of stimulus location along an auditory intensity continuum. Journal of the Experimental A nalysis of Behavior, 1966, 9, 219.225.

SaInsbury, R. S. The "feature positive effect" and simultaneous discrimination learning. Journal of Experimental Child Psychology, 1971, 11, 347-356.

(Received for publication July 30, 1976; revision accepted January 3, 1977.) 